\title{
Grundwasser in ehemaligen deutschen Steinkohlenrevieren - ein wissenschaftlicher Blickwinkel auf Grubenflutungen
}

\author{
Timo Kessler 1,3 (D) Elke Mugova ${ }^{2}$ (D) - Henning Jasnowski-Peters ${ }^{2} \cdot$ Thomas Rinder $^{3} \cdot$ Marion Stemke $^{4} \cdot$ \\ Christian Wolkersdorfer ${ }^{5}$ (D) - Sylke Hilberg ${ }^{3}$. Christian Melchers ${ }^{2} \cdot$ Wilhelm Struckmeier · Georg Wieber ${ }^{4}$. \\ Maria-Th. Schafmeister ${ }^{1}$
}

Eingegangen: 5. September 2019/ Überarbeitet: 23. April 2020 / Angenommen: 6. August 2020 / Online publiziert: 2. September 2020 (c) Der/die Autor(en) 2020

\section{Zusammenfassung}

Zum Ende des Jahres 2018 wurde in Deutschland der Steinkohlenbergbau eingestellt. Damit beginnt auch in den bis zuletzt aktiven Revieren die Phase des Nachbergbaus inklusive einer dauerhaften Wasserhaltung. Diese beinhaltet typischerweise eine teilweise Grubenflutung und die Ableitung von Grubenwasser in nahegelegene Vorfluter. Im Blickpunkt der öffentlichen und wissenschaftlichen Diskussion stehen bergbauliche Schadstoffquellen und deren Risiken für die oberflächennahen Trinkwasserressourcen. Es existieren eine Reihe Kriterien und Kontrollmechanismen, um potenziellen Schadstoffaustrag zu erkennen und zu verhindern. Dazu zählen moderne Monitoringprogramme und Modellanwendungen, welche bestehende Schadstoffprognosen verbessern können. Daneben ist auch eine rechtlich und fachlich begründete Bewertung von Gefahrenszenarios eine entscheidende Größe für nachhaltiges Risikomanagement. Der Grubenwasseranstieg eröffnet jedoch auch Chancen, ehemalige Bergwerke für energetische Nutzungen umzurüsten. Bereits heute werden Heizkraftwerke mit Grubenwasser betrieben und es besteht großes Ausbaupotenzial. Dieser Beitrag diskutiert die Chancen und Risiken von Grubenflutungen in Deutschland und gibt einen Ausblick, welche Entwicklungen und Herausforderungen in Zukunft zu erwarten sind.

Keywords Mine water $\cdot$ Hard coal mining $\cdot$ Flooding $\cdot$ Post-mining $\cdot$ Monitoring

\author{
Timo Kessler \\ timo.kessler@sbg.ac.at \\ Elke Mugova \\ elke.mugova@thga.de \\ Henning Jasnowski-Peters \\ henning.peters@thga.de \\ Thomas Rinder \\ thomas.rinder@sbg.ac.at \\ Marion Stemke \\ mstemke@uni-mainz.de \\ Christian Wolkersdorfer \\ christian@wolkersdorfer.info \\ Sylke Hilberg \\ sylke.hilberg@sbg.ac.at \\ Christian Melchers \\ christian.melchers@thga.de \\ Wilhelm Struckmeier \\ willistr@online.de
}

Georg Wieber

wieber@uni-mainz.de

Maria-Th. Schafmeister

schaf@uni-greifswald.de

1 Institut für Geographie und Geologie, Universität Greifswald, Friedr.-Ludwig-Jahn-Str. 17A, 17487 Greifswald, Deutschland

2 Research Institute of Post-Mining, Technische Hochschule Georg Agricola, Herner Straße 45, 44787 Bochum, Deutschland

3 Institut für Geographie und Geologie, Universität Salzburg, Hellbrunnerstraße 34, 5020 Salzburg, Österreich

4 Institut für Geowissenschaften, Johannes-Gutenberg-Universität Mainz, J.-J.-Becher-Weg 21, 55128 Mainz, Deutschland

5 South Africa, n Research Chair for Acid Mine Drainage Treatment, Tshwane University of Technology (TUT), Private Bag, X680, 0001 Pretoria, Südafrika 


\title{
Groundwater in former German coal mining areas-a scientific perspective on mine floodings
}

\begin{abstract}
At the end of 2018, hard coal mining in Germany was terminated. This date marks the beginning of the post-mining phase, including permanent mine water management in the abandoned coalfields. The post-mining phase typically includes partial mine flooding and the discharge of mine water into nearby receiving water courses. Public and scientific discussions have been focused on the sources of mining pollutants and potential risks for the near-surface drinking water resources. Several criteria and control mechanisms have been developed to identify and prevent potential pollutant discharge. These include modern monitoring programs and model applications that can improve existing predictions of pollutant behavior. In addition, legally and technically supported assessments of hazard scenarios are critical for sustainable risk management. Mine water rebound, however, opens opportunities to convert former mines for energy use. Combined heat and power plants can be operated with mine water and there is a great potential for expansion. This paper reviews opportunities and risks of hard-coal mine flooding in Germany and provides an outlook on what developments and challenges can be expected in the future.
\end{abstract}

\section{Einleitung}

Im Dezember 2018 wurden in Deutschland die letzten beiden aktiven Steinkohlenbergwerke Prosper-Haniel und Ibbenbüren offiziell stillgelegt (Kurkamp et al. 2019). Damit endet eine Ära, welche die deutschen Bergbauregionen unter anderem an Ruhr und Saar seit dem Mittelalter geprägt hat und zu ihren Hochzeiten Arbeit für rund 600.000 Menschen geschaffen hat (Kretschmann et al. 2017). Über Jahrhunderte war der Steinkohlenbergbau ein wichtiger Wirtschaftsfaktor in verschiedenen Regionen Deutschlands und gleichzeitig Standbein des Wirtschaftsaufschwungs nach dem Zweiten Weltkrieg. Mit dem Ende des Steinkohlenabbaus bleibt das Erbe des Bergbaus, welches die Menschen in den betroffenen Regionen in den nun folgenden Jahrhunderten begleiten wird. Dieses Erbe beinhaltet Ewigkeitsaufgaben, welche laut Ruhrkohle AG (RAG 2014) die langfristige Grubenwasserhaltung, die Sanierung kontaminierter Standorte sowie Poldermaßnahmen zur Regulierung des oberflächennahen Wasserhaushalts beinhalten (Westermann et al. 2017). Zu diesem Zweck wurde im Jahr 2007 zwischen dem Land Nordrhein-Westfalen, dem Saarland und der RAG-Stiftung der Erblastenvertrag zur Bewältigung der Ewigkeitslasten des Steinkohlenbergbaus der RAG AG nach endgültiger Einstellung des Bergbaus im Rahmen der sozialverträglichen Beendigung des subventionierten Steinkohlenbergbaus in Deutschland zum Ende des Jahres 2018 geschlossen (RAG 2014).

Seit Beginn des mitteleuropäischen Steinkohlenabbaus ist die Geschichte der Kohlenförderung eng mit jener der Wasserhaltung verknüpft, da der Abbau in größeren Tiefen ohne eine umfassende Wasserhaltung nicht möglich ist. In den großen Bergbaurevieren in Deutschland wurden Steinkohlen überwiegend im Untertagebergbau gewonnen. Mit dem fortschreitenden Bergbau und verbesserter Bergbautechnik konnten Steinkohlen aus immer tiefer liegenden Flözen gefördert werden. Um dies zu gewährleisten, wur- de das durch Klüfte und Störungen in das Grubengebäude eindringende Grundwasser (Grubenwasser) mittels Pumpen dauerhaft gehoben. Diese Maßnahmen zur Hebung und Ableitung von Grubenwasser in nahegelegene Oberflächengewässer bergen die Gefahr von Verunreinigungen. In der Regel mischen sich die meist hoch mineralisierten Tiefenwässer mit der Vorflut und lassen die Mineralisation unterstrom der Einleitung spürbar ansteigen. Dies führt dazu, dass die ökologischen Auswirkungen von gehobenem Grubenwasser in nahezu allen Betriebsphasen eines Bergwerks eine entscheidende Rolle spielen, auch noch lange nach Beendigung des aktiven Bergbaus (Kretschmann et al. 2017).

Nach der Stilllegung sollen die offenen Grubengebäude entweder kontinuierlich oder schrittweise geflutet werden, wobei auf einen weitgehend selbstregulierenden Grubenwasseranstieg abgezielt wird. Häufig werden Grubenflutungen von Monitoringprogrammen begleitet, um ökologische Auswirkungen frühzeitig erkennen zu können. Diese beinhalten klare Regelungen zu Eingreifkriterien und möglichen Maßnahmen. Die zentrale Fragestellung ist, ob und in welchem Ausmaß das Grubenwasser gehoben werden muss, um Grund- und Oberflächengewässer wirksam vor Kontaminationen zu schützen. Dazu muss unter Berücksichtigung aller wirtschaftlichen, ökologischen und technischen Aspekte ein langfristiges Grubenwasserniveau identifiziert und eingestellt werden. Für die Bergwerksbetreiber, beispielsweise an Ruhr, Saar und Ibbenbüren, ergibt sich daraus eine Ewigkeitsaufgabe, welche teils hohe Investitions- und Energiekosten für einen dauerhaften Pumpenbetrieb erforderlich machen. Dabei gilt: in Abhängigkeit vom Höhenniveau des genehmigten Grubenwasseranstieges fallen die Pumpkosten höher oder niedriger aus, weshalb an vielen ehemaligen Bergwerkstandorten wirtschaftlich und ökologisch vertretbare Grubenwasserkonzepte entwickelt und geprüft werden. Dieser Beitrag soll die wissenschaftlichen Aspekte eines wiederansteigenden Grubenwas- 
serspiegels an ehemaligen Bergwerksstandorten erläutern und diskutieren.

\section{Schadstoffquelle Grubenwasser}

\section{Chemische Beschaffenheit}

Mit dem Begriff Grubenwasser wird ,alles Wasser, das mit Tief- und Tagebauen in Kontakt steht oder stand" bezeichnet (Burghardt et al. 2017). Es handelt sich um ein Mischwasser bestehend aus Grundwasser, Formationswasser, Sickerwasser und Prozesswasser, welches in das Grubengebäude fließt und dort zentral gesammelt wird (Wohnlich et al. 2013). Saures Grubenwasser (Singer und Stumm 1970) ist eines der ökologischen Hauptprobleme im $\mathrm{Zu}$ sammenhang mit Bergbau im Allgemeinen und den meisten Steinkohlenbergbauen im Besonderen, nicht aber dem im Saarland, Ruhrgebiet und Ibbenbüren. Definitionsgemäß liegt saures Grubenwasser vor, sofern die gehobenen Wässer einen pH-Wert von kleiner 5,6 aufweisen (Blowes et al. 2014; Nordstrom 2011; Stumm und Morgan 1996; Younger et al. 2002). Ursache der Versauerung ist die Verwitterung von Disulfiden (hauptsächlich Pyrit und Markasit) in den kohleführenden Formationen. Ein Großteil der relevanten toxischen Metalle gelangt entweder direkt oder indirekt über die (Di)-Sulfidverwitterung ins Grubenwasser. Hohe Eisenkonzentrationen erhöhen das Säurebildungsvermögen von Grubenwasser durch dessen Hydrolyse. Gleichzeitig führt ein sinkender $\mathrm{pH}-$ Wert in der Regel zu einer höheren Löslichkeit und Mobilität von Metallen. Mit zunehmender Abbautiefe steigt die Mineralisation des Grubenwassers, was zur Folge hat, dass im Ruhrgebiet und in Ibbenbüren Grubenwässer mit mehreren Gramm gelöstem Salz je Liter keine Seltenheit sind (Domalski 1988; Wedewardt 1995). Stellenweise werden auch polyzyklische, aromatische Kohlenwasserstoffe (PAK) in Gewässern und Böden mit dem Steinkohlenbergbau in Zusammenhang gebracht. PAK sind natürlicher Bestandteil von Steinkohlen (Abdel-Shafy und Mansour 2016) und zeigen beispielsweise an Saar und Ruhr, wo sie auch in Gewässersedimenten vorkommen, charakteristische Muster und Konzentrationen (Achten und Hofmann 2009). Sie befinden sich vorrangig in der Suspensionsfracht und werden durch aquatischen Transport in Gewässer und Sedimente ausgetragen (Yang et al. 2008).

Neben den natürlich vorkommenden Schadstoffen sind auch originär anthropogene Verunreinigungen relevant. Insbesondere der Einsatz von Maschinen untertage und deren Betriebsmittel bergen das Risiko der Grubenwasserverunreinigung mit Kohlenwasserstoffen. Von speziellem Interesse sind die bis Mitte der 1980er-Jahre in Hydraulikölen eingesetzten polychlorierten Biphenyle (PCB) (z. B. AG „PCB
Monitoring“ 2018). Deren Ersatzstoffe, die Tetrachlorobenzyltoluene (UGILEC 141), verhalten sich ökotoxikologisch ähnlich, sodass auch mit potenziellen Verunreinigungen des Grubenwassers durch diese Komponenten gerechnet werden muss (Heim et al. 2004).

In den letzten Jahrzehnten wurden in den Bergwerken im Ruhrgebiet Reststoffe aus Müllverbrennungsanlagen und der Rauchgasentschwefelung nach Untertage gebracht und vermischt mit Zement als Versatzmaterial verwendet (Jäger et al. 1990). Dadurch ist ein höher konzentriertes Metallinventar in den Bereichen der Verfüllung vorhanden, welches bei ungünstigen physikalisch-chemischen Verhältnissen freigesetzt werden kann. Vorsorglich sollten daher in Bereichen mit untertägiger Verbringung auch die Elemente Arsen, Blei, Cadmium, Chrom, Quecksilber, Thallium und Zinn Teil der Risikoanalyse sein. Prinzipiell ist die Mobilität der einzelnen (Halb)-Metalle sehr unterschiedlich und bedarf daher aufwändiger chemisch-thermodynamischer Modellierungen. Himmelsbach und Wendland (1999) haben in Laborversuchen gezeigt, dass die Bildung von Metall-Chlorid-Komplexen in hoch mineralisierten Tiefenwässern zwar eine ideale Ausbreitung von Cadmium in strömungsaktiven Klüften erlaubt, gleichzeitig aber zu einer adsorptiven Immobilisierung von Blei führt.

\section{Geogene Einflüsse}

Im Ruhrgebiet treten hochmineralisierte Grubenwässer zutage, die einen erheblichen Anteil an Tiefenwässern haben (Wedewardt 1995). Die chemische Zusammensetzung der Wässer wird dabei durch Lösung von Mineralen (Phasen) kontrolliert und unterliegt zeitabhängigen Veränderungen durch reaktive oder mikrobielle Abbauvorgänge (Younger 1997). Beispielsweise verläuft die Oxidation von Pyrit, Markasit oder Pyrrhotin zu dreiwertigem Eisen abiotisch sehr langsam, wird aber in Gegenwart von Bakterien- und Archaeenstämmen um den Faktor $10^{6}$ beschleunigt (Evangelou und Zhang 1995). Weiters ist die Kenntnis vorhandener Mineralphasen ein Faktor für die Entschlüsselung der Grubenwassersignaturen (Nordstrom und Alpers 1999). Deren genaue Identifizierung ist selbst bei Kombination von geochemischen Elementanalysen und semiquantitativen Mineralbestimmungsmethoden mitunter schwierig und folglich bleiben Kenntnislücken bezüglich der ablaufenden Reaktionen und deren Reaktionskinetik. Beispielsweise beeinflussen Spurenelementkonzentrationen im Pyrit dessen Löslichkeit. Durch die Kenntnis des Mineralbestands im Einflussbereich eines Bergwerkes können unter Berücksichtigung der vorherrschenden Fließverhältnisse geogene Hintergrundwerte ermittelt bzw. abgeleitet werden. Diese Gesteinshintergrundwerte sind eine wichtige Information für die Bewertung von Grubenwasser, denn nicht jede Grenzwertüberschreitung in einer Bergbauregion ist 
zwangsläufig auf die Abbauaktivität zurückzuführen (Nordstrom 2015). Für das Grubenwassermanagement muss daher zwischen den anthropogenen und natürlichen Einflüssen auf die Wasserqualität unterschieden werden. In den Kohlerevieren Pennsylvanias ließ sich beispielsweise zeigen, dass die zeitliche Entwicklung des Grubenwasserchemismus in Abhängigkeit von den geologischen Rahmenbedingungen sehr unterschiedlich ausfallen kann (z.B. Burrows et al. 2015; Cravotta III 2008a, 2008b). Dabei kann der Zeitraum bis zur Verbesserung der Grubenwasserqualität nach Einstellung der Förderung zwischen wenigen Jahrzehnten und hunderten von Jahren betragen (z. B. Younger 1997; Banwart und Malmström 2001; Burrows et al. 2015).

\section{Untersuchungsmethoden bei Grubenflutungen}

\section{Modellanwendungen}

Die Hydraulik von Grubenflutungen ist in erster Linie eine hydrogeologische Konzeptvorstellung. Bei der Flutung werden die Pumpen der untertägigen Wasserhaltung in der Regel dauerhaft abgeschaltet. Daraufhin steigt das Grubenwasser durch Zulauf von Tiefengrundwasser sowie seitlichen oberflächennaher Grundwässer und Grundwasserneubildung an, und es kann sich langfristig ein natürlicher Grundwasserspiegel einstellen. Alternativ kann das Grubenwasseranstiegsniveau durch dauerhaften Tauchpumpenbetrieb kontrolliert werden, z. B. in den ehemaligen Bergwerksschächten. Die zeitliche Entwicklung des Grubenwasseranstiegs sowie das endgültige Grubenwasserniveau sind von hohem Interesse für die nachbergbauliche Grubenwasserhaltung. Bergwerksflutungen dauern meist über mehrere Jahre bis Jahrzehnte, wobei hydrostatische und chemische Effekte noch weit über den eigentlichen Wiederanstieg hinaus auftreten können (Wolkersdorfer 2008; Younger and Adams 1999). Geflutete Bergwerke stellen ein spezifisches hydraulisches System dar, welches sich aus Hohlräumen und benachbarter Gebirgsmatrix zusammensetzt. Durch die Auflast des Deckgebirges verstürzen langfristig Hohlräume und damit auch hydraulische Wegigkeiten und Verbindungen im Grubengebäude. Es empfiehlt sich daher, die Flutungsdynamik einerseits mithilfe von Monitoringprogrammen zu begleiten und aufzuzeichnen, und andererseits mittels numerischer Modelle zu prognostizieren. Modelle sind besonders für solche Standorte von Vorteil, die über kein ausreichendes Monitoring verfügen oder an denen kritische Grubenwasserspiegel nicht überschritten werden dürfen.

Im Steinkohlenbergbau wurden bislang unterschiedliche quantitative Modellierungsansätze für die Prognose des Grubenwasseranstiegs angewendet (z.B. Adams und Younger 1997; Azrag et al. 1998; Burke und Younger
2000; Gandy und Younger 2007; Kim und Choi 2018; Sherwood und Younger 1994; Toran und Bradbury 1988). Grubenflutungen unterliegen meist einer Kombination aus Matrixfluss und Hohlraumströmung. Anders als in herkömmlichen Strömungsmodellen für poröse Medien, bei denen in der Regel das laminare Darcy-Gesetz Eingang findet, ist die Wasserbewegung in Grubenhohlräumen während des Wasseranstiegs weitgehend turbulent (Wolkersdorfer 2008). Die bestehenden Modelle können basierend auf der Strömungscharakteristik in drei Ansätze unterteilt werden: (a) physikalisch motivierte Modellkonzepte, welche die Strömung zwischen Hohlräumen und Matrix differenziert berechnen (Adams and Younger 2001), (b) semi-explizite Parametermodelle (Boxmodelle), welche die Flüsse über definierte Volumina bilanzieren (Burke and Younger 2000; Choi et al. 2012; DMT GmbH \& Co. KG 2011) sowie (c) voll diskretisierte, numerische Kontinuummodelle (Abb. 1). Adams und Younger (2001) empfehlen für die Auswahl des Modellansatzes den Zeitraum sowie die Größe des zu modellierenden Reviers zu berücksichtigen. Je kleiner das Modellgebiet desto eher können Modelle angewendet werden, welche explizit den Hohlraumfluss berücksichtigen. Für Modellgebiete von $100 \mathrm{~km}^{2}$ Größe und mehr empfehlen sich semi-explizite Modelle, welche die kleinskaligen Heterogenitäten mitteln, jedoch die regionalen Strukturen im Modell abbilden können. Solche Modelle wurden typischerweise für die Simulation von Grubenwasseranstiegen eingesetzt (Gandy and Younger 2007; Kortas and Younger 2007). Für großräumigere Betrachtungen kommen in erster Linie numerische Kontinuummodelle in Betracht.

Neben dem zeitlichen Flutungsverlauf ist die hydrochemische Beschaffenheit des Grubenwassers von großer Bedeutung. Im Hinblick auf mögliche Grubenwasserübertritte in überlagernde Aquifere oder gar Oberflächengewässer ist es unabdingbar, die Transportwege und Schadstofffrachten aus dem Grubengebäude zu kennen. Auch dabei gibt es vielversprechende Modellansätze, die Schadstoffentwicklung und -menge zu prognostizieren (Banks et al. 2010; Wolkersdorfer 2008). In erster Linie versuchen solche Modelle den Ursprung, die Größenordnung und den zeitlichen Verlauf von potenziellen Schadstoffquellen zu berechnen (Adams and Younger 2001). Bei der Weiterentwicklung der numerischen Codes werden auch reaktive Transportprozesse von saurem Grubenwasser integriert (Molson et al. 2012). Aufgrund langer Aufenthaltszeiten und sich verändernder hydrochemischer Milieus werden chemische Sorptions- oder Umwandlungsprozesse im Grubengebäude begünstigt. Bei der reaktiven Transportmodellierung wird die Reaktionskinetik, d.h. die chemischen Reaktionsraten, für die individuellen Schadstoffe explizit anhand der physikalischen Randbedingungen berechnet. Das bedeutet, dass die zeitabhängigen Konzentrationsveränderungen entlang des 


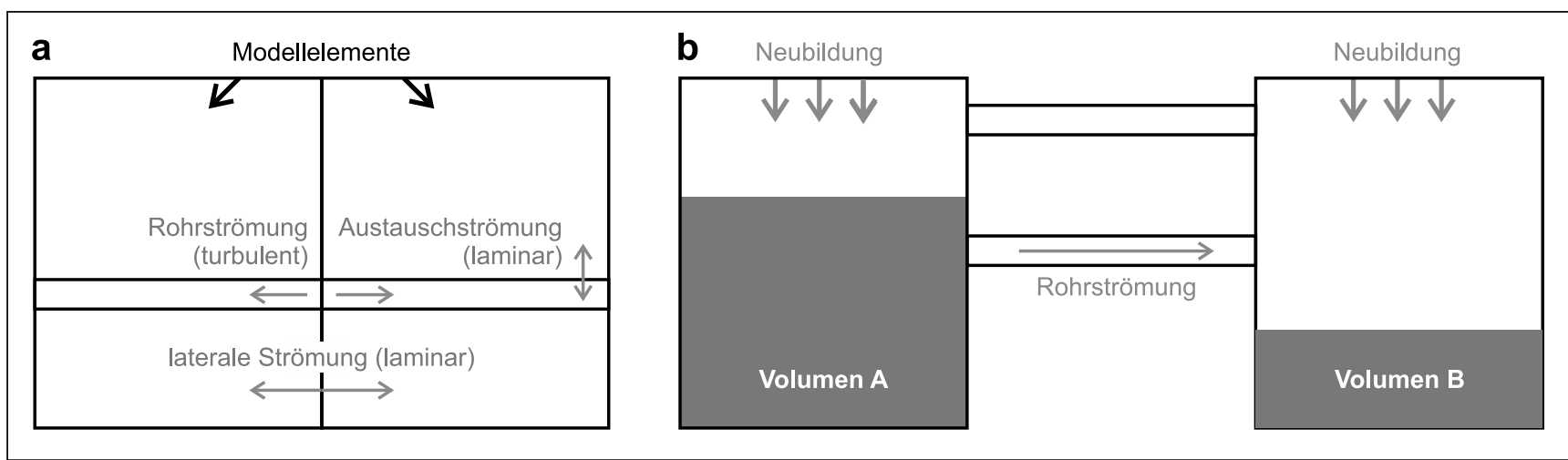

Abb. 1 Konzeptionelle Modellvorstellung von (a) einem diskreten Hohlraummodell und (b) einem Parametermodell wie dem Box Modell. In einem diskretisierten Kontinuummodell werden die Strömungstypen vergleichbar dem Hohlraummodell (a) implementiert

Fig. 1 Conceptual model of (a) a discrete void-space model and (b) a lumped-parameter model like the Box Model. In a fully-discretized continuum model the flow types implemented similar to the void-space model (a)

simulierten Transportpfades berücksichtigt werden und in die Frachtberechnungen der relevanten Schadstoffe einflieBen.

Eine Besonderheit bei der Modellierung von Strömung und Transport in Grubengebäuden ist die Ausbildung von Dichteschichtungen im Grubenwasserkörper. Erste Untersuchungen in gefluteten Bergwerken wurden 1961 von Stuart and Simpson (1961) durchgeführt, in Deutschland von Wolkersdorfer (1996) und Forschungsarbeiten für den deutschen Steinkohlenbergbau erfolgten ab Ende der 1990erJahre (Coldewey et al. 1999; Kories et al. 2004; Rüterkamp 2001). Tritt Dichteschichtung auf, bilden sich eine oder mehrere Zwischenschichten zwischen Wasserkörpern unterschiedlicher Dichte. In der Regel ist das höher mineralisierte Grubenwasser mit höherer Dichte in den tieferen Grubenbauen vorzufinden, wohingegen das Wasser mit besserer Qualität oberflächennah auftritt (Nuttall and Younger 2004; Wolkersdorfer 2008). Als natürlicher Prozess entsteht dadurch eine Barrierewirkung, welche für die Qualität des austretenden Grubenwassers von großer Bedeutung ist und so Aufwand und Kosten der Grubenwasserreinigung minimieren kann. Eine künstlich eingestellte Dichteschichtung könnte demzufolge als In-situ-Sicherungsmaßnahme von Grubenwasser genutzt werden. Dafür ist es notwendig, Fließwege des Grubenwassers zu begrenzen und eine Zirkulation des Wassers im Grubengebäude zu unterbinden, beispielsweise durch die Errichtung hydraulischer Dämme. So ist der Aufbau einer Schichtung umso wahrscheinlicher, je weniger großräumige Konvektionskreisläufe sich ausbilden (Wolkersdorfer 1996). Selbst eine künstliche Überschichtung mit Wasser mit geringerer Dichte ist als Schutzmaßnahme nach Abschluss der Flutung denkbar (Coldewey et al. 1999; Eckart et al. 2010; Kories et al. 2004; Luckner and Morgenstern 2006), sofern die großräumige Konvektion unterbunden werden kann.

\section{Isotopenchemische Analytik}

Für die Prognose von Schadstofffrachten im Zuge von Grubenflutungen sind Informationen über Konzentration, Herkunft, Transportwege und Prozesse notwendig. Isotopenuntersuchungen von Wasserproben können dabei helfen, durch Analyse der isotopenchemischen Signaturen Rückschlüsse auf Alter, Zustrom und Verweildauer zu ziehen. Balci et al. (2007) haben die stabilen Isotope des Sulfatschwefels und -sauerstoffs analysiert, um die Herkunft des Sulfats im Grubenwasser zu identifizieren. Gammons et al. (2010) haben deren Isotopensignaturen verwendet, um die Grundwasserkontamination durch Grubenwässer zu identifizieren. Ähnliches ist unter Einbeziehung der Strontiumisotope möglich (z.B. Chapman et al. 2013). Durch die Einbindung isotopengeochemischer Methoden in die Hydrogeochemie ist es unter anderem gelungen, die Genese der salinaren Wässer im Oberrheingraben zu entschlüsseln (z.B. Pauwels et al. 1993). Über die Interpretation von Spurenelementen konnten dabei Korrelationen zwischen den angetroffenen Grundwässern mit den jeweilig relevanten geologischen Schichten hergestellt werden, in denen diese Wässer gebildet wurden. Aus diesen und anderen Studien (Grobe und Machel 2002; Lüders et al. 2010) lässt sich ableiten, dass ein Multiproxyansatz notwendig ist, um die Entstehung von tiefen Grundwässern abzuleiten.

\section{Monitoring und Risikomanagement}

Eine Prognose der Anstiegs- bzw. Schadstoffkurven bei Grubenflutungen ist in der Regel mit Unsicherheiten behaftet. Daher sollte während der Grubenflutung ein Monitoring vorgesehen werden, das neben einer Kontrolle und Dokumentation ein vertieftes Verständnis der hydrogeologischen und geochemischen Rahmenbedingungen ermöglicht. Daraus werden Zusammenhänge der Wirkmechanismen und 
Tab. 1 Einwirkungspotenziale, die als Kriterien für den Entwurf von Monitoringprogrammen dienen. (Linke Spalte nach Grigo et al. 2007) Table 1 Impact potentials as criteria for the design of monitoring programs. (Left column after Grigo et al. 2007)

\begin{tabular}{ll}
\hline Kriterien der Bezirksregierung Arnsberg für das Ruhrrevier & Weitere Kriterien für Steinkohlenreviere allgemein \\
\hline $\begin{array}{l}\text { Wasserqualität, insbesondere Hydrochemie und Altlastenmobilisierung } \\
\text { (z.B. PCB) }\end{array}$ & In-situ Prozesse in Grubenbauen (Pyritoxidation, Mikrobiologie) \\
Bodenhebungen & $\begin{array}{l}\text { Fließwegbarrieren bzw. verminderte Aufnahmefähigkeit an den } \\
\text { Hauptfließwegen }\end{array}$ \\
Gasaustritte & Deckgebirgsdurchlässigkeiten (Gas/Wasser) \\
Tagesbruchgefahr bezogen auf Schächte und Abbaubereiche & Vermehrte Pumpenausfälle (Korrosion, Scaling) \\
Vernässungszonen und schwallartige Austritte im Bereich der Stollen & - \\
\hline
\end{tabular}

steuernden Maßnahmen abgeleitet, um die ökonomischen, sozialen und ökologischen Aspekte des Grubenwasseranstiegs planen zu können (Krzemień et al. 2016). Sofern Prognose und Monitoring übereinstimmen, kann dann von einer langfristigen Sicherheit während und nach der Flutung ausgegangen werden. Bei Abweichungen ist die Planung gegebenenfalls anzupassen.

Erstmals wurde bei der Flutung des Kalibergwerks Hope ein umfangreiches physiko-chemisches Monitoring in einem Untertagebergwerk durchgeführt (Herbert and Sander 1987; G.S.F. - Gesellschaft für Strahlen- und Umweltforschung 1985). Auch an Standorten im Ruhrgebiet wurden bestehende Monitoringkonzepte durch eine Auswahl an geeigneten Methoden für die nachbergbauliche Wasserhaltung getestet (Goerke-Mallet et al. 2017; Grigo et al. 2007; Klinger et al. 2013, 2012; Melchers et al. 2016). Diese Methoden müssen hinsichtlich Umsetzung, Intensität und Diversität für die jeweiligen Bergwerke adaptiert und erweitert werden. Dabei sieht das Risikomanagement aus wissenschaftlicher Perspektive für die Erkennung und Bewertung unerwünschter Ereignisse eine Beurteilung nach deterministischen und probabilistischen Maßstäben vor. Bei der Grubenflutung entstehen Gefahrenszenarien, welche sich von denen des aktiven Bergbaubetriebs deutlich unterscheiden können. Eine unkontrollierte Flutung kann beispielsweise die Gefahr von Erdfällen in Bereichen oberflächennahen Abbaus erhöhen (Deck et al. 2009). Auch Ausgasungen während der Flutung sind eine potenzielle Gefahr (Krause and Pokryszka 2013). Weiterhin kann der Grubenwasseranstieg darüber liegende Aquifere kontaminieren, die im Bereich des Absenkungstrichters liegen und eventuell Trinkund Brauchwasserversorgungen gefährden (Neymeyer et al. 2007).

Mögliche Gefahrenszenarien und die darauf einwirkenden Faktoren können beispielsweise mittels der Bow-TieMethode nach Reason $(1997,1990)$ für verschiedene Arbeitsbereiche visualisiert werden (Bourne et al. 2014; Primrose et al. 1996; Sullivan and Kandola 2003). Dieses Verfahren stellt auf der einen Seite Gefährdungen, deren Barrieren und Auslöser (Fehlerbaum) und auf der anderen Seite deren möglichen Abwehrmechanismen (Ereignisbaum) gegenüber. Das Resultat ist eine qualitative Risikoanaly- se, welche die kausalen Zusammenhänge von Fehlerquellen und Auswirkungen unerwünschter Ereignisse darstellt.

Die Gefahren und die damit verbundenen Präventionsmaßnahmen können schließlich in einer Risikomatrix bewertet werden, die Auskunft über die Eintrittswahrscheinlichkeit und das Schadensausmaß sowie deren Kosten-Nutzen-Abschätzung gibt. In Bourne et al. (2014) wurde ein risikobasierter Ansatz erstmals für den Entwurf von Mess- und Monitoringprogrammen bei CCSProjekten (Carbon Capture and Storage) angewendet. Im deutschen Steinkohlenbergbau wurde im Aachener Revier eine ähnliche Risikoabschätzung für flutungsbedingte Bodenhebungen anhand einer Klassifizierung von Einwirkungspotenzialen vorgenommen (Rosner et al. 2014). Die Bezirksregierung Arnsberg hat im Jahr 2007 auf Grundlage eines Gutachtens relevante Einwirkungspotenziale für Monitoringprogramme für das Steinkohlenrevier an der Ruhr identifiziert (Grigo et al. 2007; Tab. 1, linke Spalte). Dabei wurde der Fokus geologisch und tiefenstufenabhängig in Karbon, Deckgebirge und Vorfluter gelegt, um den Phasen eines potenziellen Grubenwasseranstiegs zu entsprechen.

Ein Flutungsmonitoring muss zahlreiche Kriterien berücksichtigen und im Hinblick auf mögliche Eskalationsszenarien (Abb. 2) geplant und ausgestattet werden. Dies bedeutet, dass eine größtmögliche räumliche Abdeckung und höchstmögliche Sensitivität (Frühwarnindikation) anzustreben sind. Weiterhin sind eine innovative Sensorik und intelligentes Datenmanagement wünschenswert, wobei das Monitoring und die Datenanalyse stets zweckmäBig und wirtschaftlich verhältnismäßig sein müssen (RAG 2014). In den Revieren der RAG AG in Nordrhein-Westfalen (Ruhr und Ibbenbüren) wird ein integrales Monitoringkonzept (Bezirksregierung Arnsberg 2020) für Grubenwasseranstiege angewendet, validiert und eine anwendbare und übertragbare Methodik für die Zukunft entwickelt (Drobniewski et al. 2018; Grigo et al. 2007; Witthaus und Drobniewski 2017).

\section{Bewertung von Grubenwasser}

Bei der Beurteilung von Grubenwässern und deren geplantem Anstieg sind in vielfältiger Weise gesetzliche Vorschrif- 


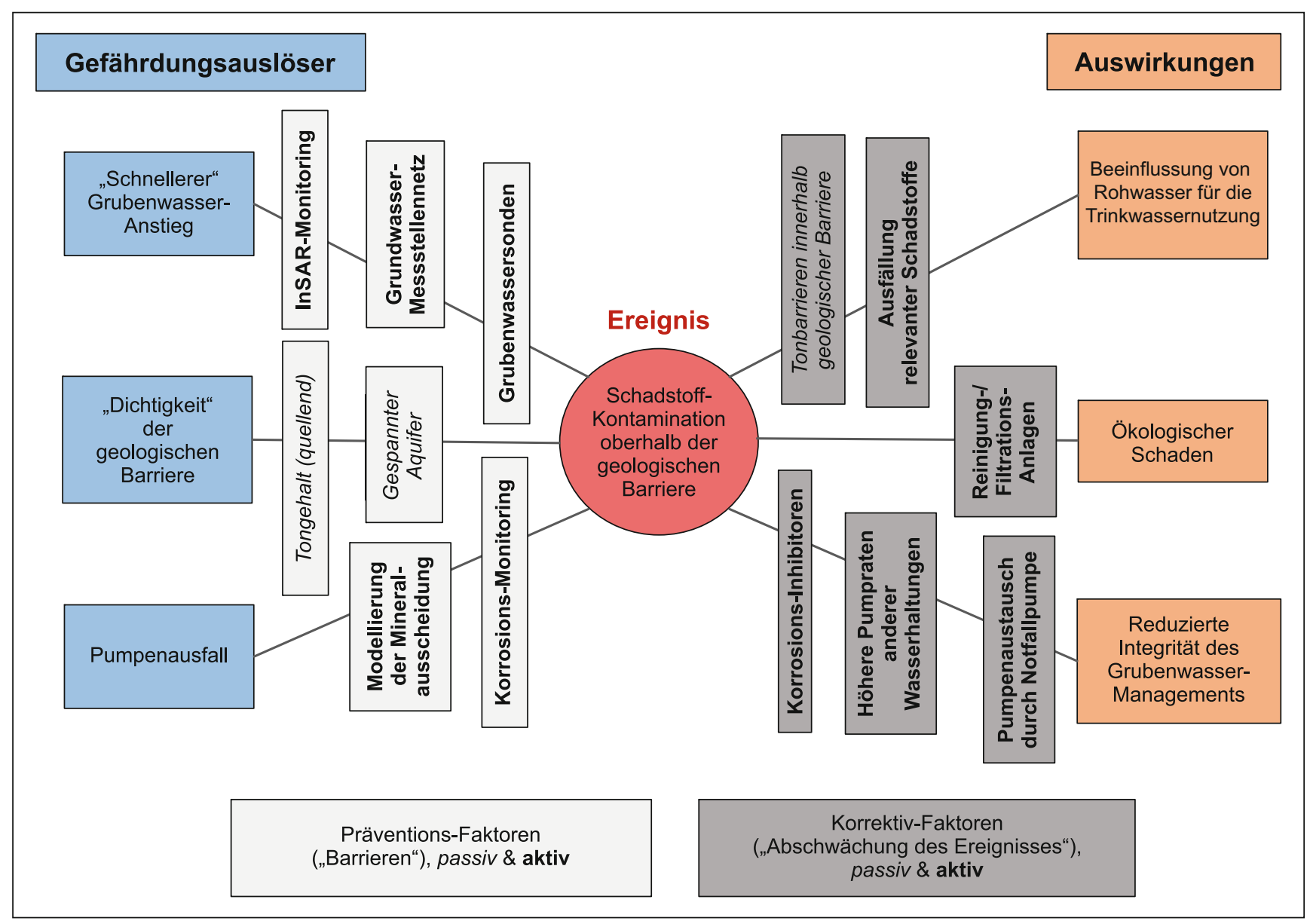

Abb. 2 Beispielhaftes Schema des Risikomanagements einer Schadstoffkontamination nach der Bow-Tie-Methode

Fig. 2 Exemplary scheme of risk management of a pollutant contamination according to the Bow-Tie method

ten zu berücksichtigen. In erster Linie betrifft dies die Einleitung von Grubenwasser in Oberflächengewässer, für die Wasserrahmenrichtlinie (EU-WRRL, WRR 2000) und Wasserhaushaltsgesetz (WHG) die Herstellung oder die Erhaltung des guten chemischen und ökologischen Zustandes fordern. Daher orientiert sich die Qualitätsbewertung im Falle des Grubenwassers an der Vorgabe, dass die Einleitung zu keiner Beeinträchtigung von Schutzgütern führen darf. $\mathrm{Zu}$ diesen gehören neben den Oberflächengewässern auch Tiere, Pflanzen, biologische Vielfalt, Boden, Stillgewässer, Grundwasser, Sachgüter und der Mensch. Typischerweise werden die jeweiligen Gefährdungen durch die vielfältigen Schadstofftypen in einer Matrix zusammengestellt, um einen raschen Überblick über die potenziell problematischen Einleitungen zu erhalten.

Für die Bewertung von Grubenwassereinleitungen sind die Vorgaben in der Oberflächengewässerverordnung OGewV (OGewV 2016) verbindlich. Maßgeblich sind die Konzentrationen im Gewässer unterstrom der Einleitstelle und nicht die des Grubenwassers selbst. Die Grenzwerte für Grubenwassereinleitungen müssen daher individuell aus den Grenzwerten für das jeweilige Gewässer, den Konzentrationen im Grubenwasser und dem Verhältnis aus Abfluss und Einleitmenge abgeleitet werden. Der pH-Wert ist beispielsweise in der OGewV als Qualitätskriterium gesetzlich geregelt, wobei je nach Gewässertyp des Vorfluters eine Wertespanne zwischen 5,5 und 8,5 für einen guten chemischen Zustand definiert ist. Das Erreichen dieses Wertes erscheint im deutschen Steinkohlebergbau derzeit nicht als Problem (Gombert et al. 2018). Mit sinkendem pH-Wert steigen jedoch in der Regel die Löslichkeit und Mobilität potenziell toxischer Metalle, wobei eine Überschreitung der Grenzwerte für einzelne Metalle auch in Grubenwasser mit zirkumneutralem $\mathrm{pH}$-Wert möglich ist. Am häufigsten lassen sich Überschreitungen anorganischer Ionen in Bezug auf die Grenzwerte der EU-Wasserrahmenrichtlinie (EU-WRRL), die OGewV oder der Orientierungswerte der Bund/Länder-Arbeitsgemeinschaft Wasser (LAWA) bei Eisen, Sulfat und Chlorid beobachten. Beispielsweise kommt es am Ibbenbürener Westfeld an den Einleitstellen regelmäßig zur Überschreitung der Grenzwerte für Chlorid, dessen Toxizität für aquatische Lebensformen zudem 
von der Gesamtwasserhärte abhängt (Elphick et al. 2011). Generell sind im deutschen Steinkohlenbergbau lokal Überschreitungen der Konzentrationen von Ammonium, Arsen, Barium, Blei, Bor, Cadmium, Chrom, Kupfer, Quecksilber und Zink bekannt (Gombert et al. 2018). Oftmals werden auch Grenzwertüberschreitungen für polyzyklische, aromatische Kohlenwasserstoffe (PAK) in Gewässern und Böden mit dem Steinkohlenbergbau in Zusammenhang gebracht. Aufgrund der weitläufigen Überschreitung der Umweltqualitätsnormen (UQN) für PAK in deutschen Oberflächengewässern sollten PAKs auch bei umfassenden Betrachtungsweisen der ökologischen Auswirkungen von Grubenwässern miteinbezogen werden.

Ein weiteres Bewertungskriterium sind die Geringfügigkeitsschwellenwerte (GFS), die von der Länderarbeitsgemeinschaft Wasser (LAWA) im Jahre 2004 festgelegt und 2017 noch einmal überarbeitet wurden (LAWA 2004). Die derzeit vorliegenden GFS basieren auf ökotoxikologischen Kriterien sowie der Trinkwasserverordnung und wurden bisher nur für oberflächennahe Grundwasserkörper ermittelt. Zudem legt die Grundwasserverordnung (GrwV) fest, dass bei Überschreitungen von Schwellenwerten, welche auf eine natürliche Ursache zurückzuführen sind, diese als eingehalten gelten und daher keine Maßnahmen zur Verminderung der Belastung erforderlich sind. Umgekehrt ist eine anthropogene Ursache in jedem Fall gegeben, sofern beispielsweise durch bergbauliche Aktivitäten eine hydraulische Verbindung zwischen tiefem und oberflächennahem Grundwasser entstanden ist. In diesem Fall müssen bei Überschreitungen von Grenzwerten Maßnahmen ergriffen werden, unabhängig von der Herkunft der Schadstoffe.

\section{Umsetzung von Grubenflutungen in Deutschland}

\section{Rechtlicher Rahmen}

In der Bundesrepublik Deutschland stellt die Grubenwasserhaltung, das heißt das Heben und Ableiten von Grubenwasser, eine Gewässerbenutzung dar und ist daher genehmigungspflichtig. Zusätzlich kommen die Umleitung von Grundwasser ( 9 , Abs. 2, Nr. 1, Wasserhaushaltsgesetz, WHG 2009) sowie die nachteilige Veränderung der Wasserbeschaffenheit ( 9 , Abs. 2, Nr. 2, Wasserhaushaltsgesetz, WHG 2009) als genehmigungspflichtige Maßnahme in Betracht. Die Genehmigungen werden durch die Bergbehörden der Länder erteilt (Beckmann 2010), haben allerdings im Einvernehmen mit der zuständigen Wasserbehörde zu erfolgen. Sämtliche Maßnahmen im Zusammenhang mit der Grubenwasserhaltung sind Teil der zugelassenen bergbaulichen Betriebspläne, die für die Errichtung, den Betrieb und für die Stilllegung des Bergwerkes verpflichtend sind ( $\$ 53$,
Abs. 1, Satz 2 des Bundesberggesetzes, BBergG 1980). Im Rahmen des bergrechtlichen Zulassungsverfahrens ist zu prüfen, ob durch die Grubenwasserhaltung schädliche Einflüsse, auch in Bezug auf Gewässerverunreinigungen, ausgeschlossen werden können (Jordan und Welsing 2017). Je nach Beschaffenheit der Grubenwässer kann das Genehmigungsverfahren eine Nachbehandlung sowie ein Monitoringprogramm für die Wasserhaltung vorschreiben. Die Formulierung von Auflagen unterliegen dem Grundsatz der Verhältnismäßigkeit und müssen daraufhin geprüft werden. Zusätzlich sind für die Hebung von Grubenwasser wasserrechtliche Erlaubnisse sowohl für das Zutagefördern des Grundwassers ( 99 Absatz 1, Nr. 5 Wasserhaushaltsgesetz, WHG 2009) als auch für die Einleitung in Oberflächengewässer (§ 9 Absatz 1, Nr. 4, Wasserhaushaltsgesetz, WHG 2009) erforderlich. Grundsätzlich legt das Wasserhaushaltsgesetz als Bewirtschaftungsziel das Erreichen eines guten ökologischen und chemischen sowie eines guten mengenmäßigen Zustands der Gewässer fest. Abweichungen von diesen Zielen sind nach $§ 30$ WHG Ziff. 1 zulässig, wenn „die Gewässer durch menschliche Tätigkeiten so beeinträchtigt oder ihre natürlichen Gegebenheiten so beschaffen sind, dass die Erreichung der Ziele unmöglich ist oder mit unverhältnismäßig hohem Aufwand verbunden wäre“ (Wieber 2013).

\section{Beispiele ausgewählter Bergwerksstandorte}

Neben den derzeit aktuell zur Flutung anstehenden Steinkohlenrevieren im Saarland, im Ruhrgebiet und in Ibbenbüren wurden in Deutschland bereits weitere Grubenreviere geflutet oder unterliegen derzeitig der Flutung. Ohne besondere Reihung betrifft dies unter anderem das Aachener Revier (NRW, Belgien), das Döhlener Bergbaurevier (Freital, Sachsen), das Ibbenbürener Steinkohlenrevier (NRW, Westfeld), das Lugau-Oelsnitzer Steinkohlenrevier (Sachsen), das Rheinisch-Westfälische Steinkohlerevier (NRW), das Saarrevier (Saarland, Frankreich), das Zwickauer Steinkohlenrevier (Sachsen), sowie die Reviere Stockheim und die Bayerische Pechkohlenmulde in Bayern. Im Aachener Revier und im anschließenden Bereich auf der niederländischen Seite ist die Flutung bereits abgeschlossen, ohne dass bislang negative Auswirkungen auf das Grundwasser oder Vorfluter bekannt geworden sind (Heitfeld et al. 2002; Rosner 2011; Wolkersdorfer und Bowell 2005). Gleiches gilt für das Stockheimer Revier und die Bayerische Pechkohlenmulde. In beiden Fällen gibt es nur jeweils einen Grubenwasseraustritt, der zu kleinräumiger lokaler Beeinträchtigung des Vorfluters führt, aber nach wenigen hundert Metern durch Verdünnung nicht mehr erkennbar ist (Wolkersdorfer und Bantele 2013). In Zwickau ist die Flutung bereits seit über 20 Jahren abgeschlossen. Einzelne diffuse Grubenwasseraustritte sind an der Geländeoberfläche fest- 
zustellen. Für das Grubenwasser, welches geothermisch genutzt wird, erfolgt unter Kontrolle der physiko-chemischen Wasserparameter die Einleitung in den Vorfluter Zwickauer Mulde (Hoth 2008; Röder 2018). Im Ibbenbürener Westrevier sind die Tiefbaue seit langem geflutet und nach passiver und aktiver Reinigung wird das austretende Grubenwasser der Vorflut Ibbenbürener Aa zugeleitet (Goerke-Mallet und Drobniewski 2016). Die Steinkohlenbergwerke des Saarreviers (Lothringen) auf französischer Seite sind vollständig geflutet und mittels eines untertägigen hydraulischen Damms von den noch nicht gefluteten Bergwerken auf deutscher Seite abgeriegelt. Eine Besonderheit stellt das sächsische Revier Lugau-Oelsnitz dar. Obwohl seit Stilllegung des Bergbaus im Jahr 1971 die Flutung begann, erfolgt der Anstieg des Grubenwassers nur sehr langsam. Ein erstes Austreten des Grubenwassers an der Tagesoberfläche wird um das Jahr 2033 erwartet (Felix et al. 2010a).

In den meisten ehemaligen deutschen Steinkohlenrevieren erfolgt eine direkte Einleitung des gehobenen Grubenwassers in die Vorflut. Im Döhlener Bergbaurevier in Freital/Sachsen gibt es beispielsweise keine Überschreitung relevanter Grenzwerte der wasserrechtlichen Erlaubnis (Goerke-Mallet et al. 2016). Auch im Aachener Revier und im Saarland wird das gehobene Grubenwasser bislang ohne vorgeschaltete Grubenwasserreinigung in die Vorflut eingeleitet (MUV 2017). Gegenwärtig wurden eine Reihe von Maßnahmen zur Ermittlung der Herkunft sowie der Reduzierung der Chlorid-, Wärme-, Ammonium- und PCBBelastung in das Programm des 2. Bewirtschaftungszyklus aufgenommen, die verpflichtend bis Ende 2021 umgesetzt werden müssen (MUV 2017). Im rheinisch-westfälischen Revier wurde vor Abwerfen der Grube Auguste Victoria eine untertägige Bariumabscheidung betrieben, und am Standort Friedlicher Nachbar im selben Revier wurde das Wasser einer Eisenfällung unterzogen. Das Grubenwasser wird dabei zunächst über Kaskaden belüftet und mit Sauerstoff angereichert. In nachgeschalteten Absetzbecken kommt es zur Ausfällung und Abscheidung des Eisens, bevor das Grubenwasser in einem offenen Gerinne in den Vorfluter eingeleitet wird (Rahm 2018). Punktuell treten bei Grubenwasseraustritten Geruchsbelästigungen durch mikrobiologisch gebildeten Schwefelwasserstoff auf, beispielsweise an den Standorten Haus Aden und Robert Müser (Harpener Teiche), sowie im Saarland am Standort Camphausen. Mittels einer „Impfung“ der Wässer mit Wasserstoffperoxid wird Schwefelwasserstoff in elementaren Schwefel und nach der Einleitung in Sulfat überführt und damit der starke Geruch reduziert (RAG 2010). Bei allen anderen Grubenwasseraustrittsstellen im rheinisch-westfälischen Revier wird zum jetzigen Zeitpunkt keine Grubenwasserreinigungsanlage betrieben. Von 2007 bis 2010 wurde jedoch am Standort Concordia eine Pilotanlage zur aktiven und passiven Behandlung von Grubenwasser getestet, wozu unter anderem Fällungsreaktionen von Eisen, Barium und Mangan, sowie Belüftungsversuche und Sedimentation zählen (DMT 2010). Im Revier Ibbenbüren wird bereits heute Grubenwasser sowohl passiv als auch aktiv gereinigt. Wasser aus dem Ostfeld des Bergwerkes durchfließt in Püsselbüren mehrere Absetzbecken (Sedimentationsteiche Püsselbüren) und ein angeschlossenes Feuchtgebiet, bevor es über den Stollenbach in die Ibbenbürener Aa eingeleitet wird. Die Absetzbecken verringern die Fließgeschwindigkeit und es kommt zur Sedimentation des Schwebstoffgehalts. Zudem wird durch den Sauerstoffeintrag die Hydrolyse des Eisens begünstig, das in den Sedimentationsbecken ausfällt und wodurch eine bessere Einleitqualität des Grubenwassers erreicht wird. Neben dieser passiven Grubenwasserreinigung findet mit dem Wasser aus dem Westfeld am Standort Gravenhorst eine aktive Eisenfällung durch die Zugabe von Kalkmilch und zusätzlicher Belüftung statt (Rahm 2018). Weiterhin kommt es zur Flockung, Koagulation und schließlich Fällung des Eisenoxihydrats (Wolkersdorfer 2020). In Lugau-Oelsnitz zeigen Untersuchungen des Grubenwassers, dass vermutlich hoch mineralisierte Tiefenwässer austreten werden und möglicherweise eine Grubenwasserreinigung notwendig sein wird (Felix et al. 2010a). Aufgrund der hohen Eisen- und Mangankonzentrationen im Grubenwasser wird gegenwärtig die Anschaffung einer mobilen Grubenwasserreinigungsanlage diskutiert (Sächsisches Landesamt für Umwelt, Landwirtschaft und Geologie 2018). Auch im Fall einer zukünftigen geothermischen Nutzung des Wassers müsste eine Reinigung konzipiert werden (Felix et al. 2010b).

\section{Energetische Nutzungspotenziale}

Im Zeitalter der Energiewende und des Klimaschutzes kommt auch den energetischen Nutzungspotenzialen im Nachbergbau eine gewisse Bedeutung zu. Diese umfasst in erster Linie die Nutzung des Grubenwassers als geothermale Wärmequelle oder als Speichermöglichkeit für die regionale Wärme- und Stromversorgung (Grab et al. 2018). Bei der Wärmeproduktion wird zwischen zwei Systemen der Niedrig-Enthalpie-Wärmenutzung unterschieden: (a) geschlossene Wärmesonden-Kreisläufe und (b) offene Systeme, welche die Wärmeenergie unmittelbar aus dem Grubenwasser beziehen. In einer Potenzialstudie vom Landesamt für Natur, Umwelt und Verbraucherschutz Nordrhein-Westfalen (Bracke et al. 2018) wurde eine erste Abschätzung des Gesamtwärmepotenzials getroffen, das durch Grubenwasser in den Steinkohlen- und Erzrevieren zur Verfügung stehen würde. Für das Jahr 2035 steht dieser zufolge ein theoretisches Potenzial von $2186 \mathrm{GWh} / \mathrm{a}$ zur Verfügung. In NRW befinden sich derzeit zwei Heizkraftwerke, welche Wärme aus Grubenwasser beziehen: die Kraftwerke in Bochum-Werne und Essen, die seit 
2012 bzw. 2010 in Betrieb sind und Grubenwasser aus Teufen von 570 bzw. $1200 \mathrm{~m}$ mit bis zu 3001/s fördern. Mittlerweile hat das Konsortium „Grubenwasser-Ruhr“ drei weitere Grubenwasserprojekte für die Städte Essen (Bergwerk Amalie), Bergkamen (Bergwerk Haus Aden) und Bochum (Bergwerk Robert Müser) identifiziert, die sich in der planerischen Umsetzung befinden (Reiners et al. 2018). Entscheidender Faktor für den Ausbau der geothermischen Grubenwassernutzungen sind, neben der begleitenden Technologie, die rechtlichen Statuten wie das Erneuerbare-Energie-Gesetz (EEG, EEG 2017), welches die anvisierte Dezentralisierung der Energiesysteme regelt. Bei geschlossenen Systemen sind zudem die thermophysikalischen Parameter wie die Wärmeleitfähigkeit, Wärmekapazität und Wärmestromdichte ausschlaggebend für eine dauerhafte und grundlastfähige Wärmeversorgung. Technisch gesehen ist die Frage der Korrosion oder der Bildung von Ablagerungen (,Scaling“) für die Langlebigkeit geothermischer Nutzungen von erheblichem praktischen Interesse. Vor diesem Hintergrund wurde die hydrochemische Zusammensetzung von Grubenwasser und dessen Auswirkungen auf die geothermische Infrastruktur an verschiedenen Standorten studiert (Boch et al. 2017; Bozau et al. 2015; Corsi 1986; Eggeling et al. 2013; Wanner et al. 2017). Eine weitere Möglichkeit der energetischen Nutzung von Grubengebäuden ist der Umbau zu Pumpspeicherkraftwerken. Auch dabei wird zwischen geschlossenen und offenen Systemen unterschieden. Ersteres ist von Vorteil, wenn hochsalinare Tiefen- oder Grubenwässer zu erwarten sind und eine Vermischung mit Injektionswasser und dessen schleichender Versalzung vermieden werden soll. Entsprechend sind separate Leitungen und unterirdische Kavernen notwendig (Niemann et al. 2013; Perau et al. 2012). Eine 200-MW-Anlage mit einer Kapazität von 835 MWh wurde für den Standort Prosper-Haniel in Bottrop konzipiert. Die vier bestehenden Schachtanlagen werden als Zugang zur Tiefe genutzt, die unterirdischen Speicherbecken jedoch als langgestreckter Ringspeicher neu aufgefahren (Niemann et al. 2018; Niemann 2018). Im offenen System können alle im Untergrund vorhandenen Hohlräume wie Stollen, Strecken, verstürzte Abbaubereiche sowie das zur Verfügung stehende Porenvolumen genutzt werden. Es besteht daher großes Speicherpotenzial, und allein über die Nutzung der Grubenwasserhaltung könnte ein geschätztes Speichervolumen von etwa $3 \cdot 10^{6} \mathrm{~m}^{3}$ zur Verfügung stehen (Niemann et al. 2013).

\section{Ausblick und Forschungsziele}

In den vergangenen Jahren wurden für den Rückbau und die geplante Flutung von stillgelegten Steinkohlenbergwerken eine Vielzahl von Gutachten und Konzepten ausgear- beitet (z. B. RAG 2014; Wagner 2017). Diese sind notwendig, um verlässliche Prognosen für den Grubenwasseranstieg an ehemaligen Standorten erstellen zu können. Dabei stützen sich die Aussagen hauptsächlich auf Konzepte, Ergebnisse aus Beweissicherungen und numerische Berechnungen. Es existieren jedoch relativ wenige Erfahrungswerte $\mathrm{zu}$ den hydraulischen und hydrochemischen Prozessen während und nach den Bergwerksflutungen. Die Ergebnisse sind meist schlecht übertragbar und die gefluteten Bergwerke ohnehin unzugänglich. Forschungsbedarf ergibt sich insbesondere in den Bereichen Flutungs- und Schadstoffprognose, Design von Monitoringkonzepten und Erarbeitung rechtlicher Bewertungsgrundlagen für Grubenwasser. Numerische Kontinuummodelle ermöglichen die Kombination von Matrix- und Hohlraumströmung und versuchen, die Prognosen der bisher häufig angewendeten Boxmodelle zu erweitern. Daneben bedarf es moderner Monitoringmaßnahmen mit begleitender Analytik, um Schwachstellen in Grubenwasserkonzepten zu identifizieren, künftige Entwicklungen zu erleichtern und einen Beitrag zur besseren Bewältigung der zukünftigen Grubenwasserhaltung zu liefern. Neben den angewandten Fragestellungen der Grubenwasserhaltung sind auch eine Reihe verknüpfter Aspekte der Grundlagenforschung wie die Evolution mineralisierter Tiefenwässer, der reaktive Stofftransport oder die Dichteschichtungen in gefluteten Grubengebäuden Gegenstand künftiger Forschungsvorhaben. Beispielsweise ist die Reaktionskinetik von Schadstoffen in Grubenwässern abhängig von den Zusammensetzungen der Mineralphasen, welche jedoch für Kohlenbergwerke in der Regel schlecht dokumentiert sind. Auch katalytische Oxidationen von Pyrit und Markasit sind durch fehlende Daten zu Existenz und Aktivität von mikrobiellen Gemeinschaften in großer Tiefe weitgehend unerforscht. Isotopensignaturen könnten Aufschluss auf die Herkunft von Grubenwasser geben und tragen dazu bei, Quellen, Milieus und Pfade der Mineralisierung $\mathrm{zu}$ identifizieren und $\mathrm{zu}$ lokalisieren. Inwieweit die mineralisierten, tiefen Grubenwässer an der Oberfläche austreten, hängt auch von den hydrodynamischen Verhältnissen innerhalb des gefluteten Grubengebäudes ab. Durch experimentelle Untersuchungen kann versucht werden die Dynamik von Konvektionszellen und die Ausbildung von Dichteschichtungen zu entschlüsseln. Dieser Aspekt könnte letztlich auch der angewandten Forschung von Nutzen sein, indem beispielsweise Dichteschichtungen als In-situSicherungsmaßnahme in Grubengebäuden eingestellt werden könnten.

Danksagung Für die Manuskripterstellung bedanken wir uns bei den beiden Gutachtern für die konstruktiven Hinweise.

Förderung Wir danken der Ruhrkohle AG für die Unterstützung der Forschungsarbeiten des „Forum Bergbau und Wasser“ sowie dem Deutschen Stiftungszentrum für die sorgsame Mittelverwaltung. 
Funding Open Access funding provided by Projekt DEAL.

Open Access Dieser Artikel wird unter der Creative Commons Namensnennung 4.0 International Lizenz veröffentlicht, welche die Nutzung, Vervielfältigung, Bearbeitung, Verbreitung und Wiedergabe in jeglichem Medium und Format erlaubt, sofern Sie den/die ursprünglichen Autor(en) und die Quelle ordnungsgemäß nennen, einen Link zur Creative Commons Lizenz beifügen und angeben, ob Änderungen vorgenommen wurden.

Die in diesem Artikel enthaltenen Bilder und sonstiges Drittmaterial unterliegen ebenfalls der genannten Creative Commons Lizenz, sofern sich aus der Abbildungslegende nichts anderes ergibt. Sofern das betreffende Material nicht unter der genannten Creative Commons Lizenz steht und die betreffende Handlung nicht nach gesetzlichen Vorschriften erlaubt ist, ist für die oben aufgeführten Weiterverwendungen des Materials die Einwilligung des jeweiligen Rechteinhabers einzuholen.

Weitere Details zur Lizenz entnehmen Sie bitte der Lizenzinformation auf http://creativecommons.org/licenses/by/4.0/deed.de.

\section{Literatur}

Abdel-Shafy, H.I., Mansour, M.S.M.: A review on polycyclic aromatic hydrocarbons: source, environmental impact, effect on human health and remediation. Egypt. J. Pet. 25(1), 107-123 (2016). https://doi.org/10.1016/J.EJPE.2015.03.011

Achten, C., Hofmann, T.: Native polycyclic aromatic hydrocarbons $(\mathrm{PAH})$ in coals-A hardly recognized source of environmental contamination. Sci. Total Environ. 407(8), 2461-2473 (2009). https://doi.org/10.1016/J.SCITOTENV.2008.12.008

Adams, R., Younger, P.L.: Simulation of groundwater rebound in abandoned mines using a physically based modelling approach. In: IMWA 6th Int. Mine Water Assoc. Congress Bled. S. 353-362. (1997)

Adams, R., Younger, P.L.: A strategy for modeling ground water rebound in abandoned deep mine systems. Ground Water (2001). https://doi.org/10.1111/j.1745-6584.2001.tb02306.x

AG „PCB-Monitoring“: Bericht der AG „PCB-Monitoring“ nach Beendigung des Sondermessprogramms 2016-2017 - Ergebnisse der chemischen und ökotoxikologischen Analytik von Grubenwässern und von Grubenwasser beaufschlagten kleineren Bächen rechts der Saar. Report Nr. PCB-2018-01. Saarländisches Landesamt für Umwelt- und Arbeitsschutz (LUA), Saarbrücken, S. 1-89 (2018)

Azrag, E.A., Ugorets, V.I., Atkinson, L.C.: Use of a finite element code to model complex mine water problems. In: Mine Water and Environmental Impacts, Bd. 1, S. 31-41. International Mine Water Association, Johannesburg (1998)

Balci, N., Shanks III, W.C., Mayer, B., Mandernack, K.W.: Oxygen and sulfur isotope systematics of sulfate produced by bacterial and abiotic oxidation of pyrite. Geochim. Cosmochim. Acta 71(15), 3796-3811 (2007). https://doi.org/10.1016/j.gca.2007.04.017

Banks, D., Frolik, A., Gzyl, G., Rogoż, M.: Modeling and monitoring of mine water rebound in an abandoned coal mine complex: Siersza Mine, Upper Silesian Coal Basin, Poland. Hydrogeol. J. 18(2), 519-534 (2010). https://doi.org/10.1007/s10040-009-0534-z

Banwart, S.A., Malmström, M.E.: Hydrochemical modelling for preliminary assessment of minewater pollution. J. Geochem. Explor. 74(1-3), 73-97 (2001). https://doi.org/10.1016/S03756742(01)00176-5

BBergG: Bundesberggesetz i.d.F. vom 13. August 1980. Bundesberggesetz, Deutschland (1980)

Beckmann, M.: Rechtliche Rahmenbedingungen der Abschlussbetriebsplanung. In: Kühne, G., Ehricke, U. (Hrsg.) Bergrecht zwischen Tradition und Moderne: Zivil- und öffentlichrechtliche
Probleme des (ausgehenden) Steinkohlenbergbaus - Neue untertägige Nutzungsformen und -kollisionen, S. 169-197. Nomos, Baden-Baden (2010)

Bezirksregierung Arnsberg: Integrales Monitoring für den Grubenwasseranstieg in NRW Konzeptbeschreibung. Bezirksregierung Arnsberg, Dortmund (2020)

Blowes, D.W., Ptacek, C.J., Jambor, J.L., Weisener, C.G., Paktunc, D., Gould, W.D., Johnson, D.B.: The geochemistry of acid mine drainage. In: Turekian, H.D., Holland, K.K. (Hrsg.) Treatise on Geochemistry, S. 131-190. Elsevier, Oxford (2014) https://doi.org/10. 1016/B978-0-08-095975-7.00905-0

Boch, R., Leis, A., Haslinger, E., Goldbrunner, J.E., Mittermayr, F., Fröschl, H., Hippler, D., Dietzel, M.: Scale-fragment formation impairing geothermal energy production: interacting $\mathrm{H}_{2} \mathrm{~S}$ corrosion and $\mathrm{CaCO}_{3}$ crystal growth. Geotherm. Energy 5(1), 1-19 (2017). https://doi.org/10.1186/s40517-017-0062-3

Bourne, S., Crouch, S., Smith, M.: A risk-based framework for measurement, monitoring and verification of the Quest CCS Project, Alberta, Canada. Int. J. Greenh. Gas Control. 26, 109-126 (2014). https://doi.org/10.1016/J.IJGGC.2014.04.026

Bozau, E., Häußler, S., van Berk, W.: Hydrogeochemical modelling of corrosion effects and barite scaling in deep geothermal wells of the North German Basin using PHREEQC and PHAST. Geothermics 53, 540-547 (2015). https://doi.org/10.1016/j.geothermics. 2014.10.002

Bracke, R., Bussmann, G., Eicker, T., Ignacy, R., Jagert, F., DanowskiBuhren, C., Schmidt, B.: Potenzialstudie warmes Grubenwasser LANUV Fachbericht 90. Landesamt für Natur, Umwelt und Verbraucherschutz Nordrhein-Westfalen (LANUV), Recklinghausen, S. 1-154 (2018)

Burghardt, D., Coldewey, W.G., Melchers, C., Meßer, J., Paul, M., Walter, T., Wesche, D., Westermann, S., Wieber, G., Wisotzky, F., Wolkersdorfer, C.: Glossar Bergmännische Wasserwirtschaft. Fachsektion Hydrogeologie in der DGGV, Neustadt/Wstr. (2017)

Burke, S.P., Younger, P.L.: Groundwater rebound in the South Yorkshire coalfield-A first approximation using the GRAM model. Q. J. Eng. Geol. Hydrogeol. 33(2), 149-160 (2000). https://doi.org/ 10.1144/qjegh.33.2.149

Burrows, J.E., Peters, S.C., Cravotta III, C.A.: Temporal geochemical variations in above-and below-drainage coal mine discharge. Appl. Geochem. 62, 84-95 (2015). https://doi.org/10.1016/j. apgeochem.2015.02.010

Chapman, E.C., Capo, R.C., Stewart, B.W., Hedin, R.S., Weaver, T.J., Edenborn, H.M.: Strontium isotope quantification of siderite, brine and acid mine drainage contributions to abandoned gas well discharges in the Appalachian Plateau. Appl. Geochem. 31, 109-118 (2013). https://doi.org/10.1016/j.apgeochem.2012.12. 011

Choi, Y., Baek, H., Cheong, Y.-W., Shin, S.-H., Kim, G.-M., Kim, D.H.: GRAM model analysis of groundwater rebound in abandoned coal mines. Tunn. Undergr. Space Technol. 22, 373-382 (2012). https://doi.org/10.7474/TUS.2012.22.6.373

Coldewey, W.G., Hewig, R., Richter, R., Rüterkamp, P., Wedewart, M.: Mittelfristige Entwicklung des Chemismus und der Dichteschichtungen von Grubenwässern in Bergwerken und ihre Auswirkungen auf nutzbares Grund- und Oberflächenwasser. Deutsche Mon$\tan$ Technologie GmbH, Essen (1999)

Corsi, R.: Scaling and corrosion in geothermal equipment: problems and preventive measures. Geothermics 15(5), 839-856 (1986). https://doi.org/10.1016/0375-6505(86)90097-0

Cravotta III, C.A.: Dissolved metals and associated constituents in abandoned coal-mine discharges, Pennsylvania, USA. Part 1: Constituent quantities and correlations. Appl. Geochem. 23(2), 166-202 (2008a). https://doi.org/10.1016/j.apgeochem.2007.10. 003

Cravotta III, C.A.: Dissolved metals and associated constituents in abandoned coal-mine discharges, Pennsylvania, USA. Part 2: geo- 
chemical controls on constituent concentrations. Appl. Geochem. 23(2), 203-226 (2008b). https://doi.org/10.1016/j.apgeochem. 2007.10.003

Deck, O., Verdel, T., Salmon, R.: Vulnerability assessment of mining subsidence hazards. Risk Anal. 29(10), 1381-1394 (2009). https://doi.org/10.1111/j.1539-6924.2009.01238.x

DMT GmbH \& Co. KG: Abschlussbericht zum Forschungsvorhaben Optimierung von Grubenwasseraufbereitung durch Kombination technischer und passiver Verfahren. RAG Deutsche Steinkohle, Herne (2010)

DMT GmbH \& Co. KG: BoxModel Concept: ReacFlow3D—Modelling of the Flow of Mine Water and Groundwater, Mass and Heat Transport. DMT GmbH \& Co. KG, Essen (2011)

Domalski, R.F.: Bergmännische Wasserwirtschaft der Steinkohlenbergwerke Preussag AG Kohle, Ibbenbüren und Gewerkschaft Sophia-Jacoba/Hückelhoven - ein Vergleich. Westf. Berggewerkschaftskasse, Bochum, S. 60 (1988)

Drobniewski, M., Karus, C., Löchte, J.: Retreat to the future: shaping the post-mining era. Mine Water Environ 37(4), 866-867 (2018). https://doi.org/10.1007/s10230-018-0565-6

Eckart, M., Rüterkamp, P., Klinger, C., Kories, H., Gzyl, G.: Qualitätsentwicklung der Grubenwässer bei der Flutung von Steinkohlenund Erzbergwerken. Merkel, B.J. \& Schipek, M. (eds.): Grubenwässer - Herausforderungen und Lösungen, 61. Berg- und Hüttenmännischer Tag, TU BA Freiberg, S. 123-131 (2010)

EEG: Gesetz für den Ausbau erneuerbarer Energien. Bundesministerium der Justiz und für Verbraucherschutz, Berlin (2017)

Eggeling, L., Genter, A., Kölbel, T., Münch, W.: Impact of natural radionuclides on geothermal exploitation in the Upper Rhine Graben. Geothermics 47, 80-88 (2013). https://doi.org/10.1016/j. geothermics.2013.03.002

Elphick, J.R.F., Bergh, K.D., Bailey, H.C.: Chronic toxicity of chloride to freshwater species: effects of hardness and implications for water quality guidelines. Environ. Toxicol. Chem. 30(1), 239-246 (2011). https://doi.org/10.1002/etc.365

Evangelou, V.P., Zhang, Y.L.: A review: pyrite oxidation mechanisms and acid mine drainage prevention. Crit Rev Environ Sci Technol 25(2), 141-199 (1995). https://doi.org/10.1080/ 10643389509388477

Felix, M., Eckart, M., Görne, S.: Das Grubenwasseranstiegsmodell im Steinkohlerevier Lugau/Oelsnitz. Geoprofil 13, 99-104 (2010a)

Felix, M., Möllmann, G., Wagner, S., Görne, S.: Zur geothermischen Nutzbarkeit des Grubenwassers im Bergbaurevier Lugau/ Oelsnitz. Geoprofil 13, 169-175 (2010b)

G. S. F. - Gesellschaft für Strahlen- und Umweltforschung: Untersuchungen endlagerrelevanter Vorgänge vor, während und nach der Flutung des Kalisalzbergwerkes Hope. 1. Bericht. gsf-Bericht 85(12), 286 (1985)

Gammons, C.H., Duaime, T.E., Parker, S.R., Kennelly, P.: Geochemistry and stable isotope investigation of acid mine drainage associated with abandoned coal mines in central Montana, USA. Chem. Geol. 269(1/2), 100-112 (2010). https://doi.org/10.1016/ J.CHEMGEO.2009.05.026

Gandy, C.J., Younger, P.L.: Predicting groundwater rebound in the South Yorkshire Coalfield, UK. Mine Water Environ. 26, 70-78 (2007). https://doi.org/10.1007/s10230-007-0153-7

Goerke-Mallet, P., Drobniewski, M.: Planning long-term mine-water management for the Ibbenbüren coal basin. Veröff. Dt. BergbauMus. Bochum, Bd. 212., S. 108-113 (2016)

Goerke-Mallet, P., Reker, B., Westermann, S., Melchers, C.: Nachbergbau in Großbritannien: das Steinkohlenrevier South Yorkshire. Markscheidewesen 124(1), 13-21 (2017)

Goerke-Mallet, P., Westermann, S., Melchers, C.: Die Verwahrung der Bergwerke im Döhlener Becken durch die Wismut GmbH - Eine Evaluierung des Grubenwasseranstiegsprozesses. In: 17. Geokinematischer Tag des Institutes für Markscheidewesen und Geodäsie, S. 123-130. TU Bergakademie, Freiberg (2016)
Gombert, P., Sracek, O., Koukouzas, N., Gzyl, G., Valladares, S.T., Frączek, R., Klinger, C., Bauerek, A., Areces, J.E.Á., Chamberlain, S., Paw, K., Pierzchała, Ł.: An overview of priority pollutants in selected coal mine discharges in Europe. Mine Water Environ. 38(1), 16-23 (2018). https://doi.org/10.1007/s10230-019-006073

Grab, T., Storch, T., Groß, U.: 17. Energetische Nutzung von Grubenwasser aus gefluteten Bergwerken. In: Bauer, M., Freeden, W., Jacobi, H., Neu, T. (Hrsg.) Handbuch Oberflächennahe Geothermie, S. 523-586. Springer, Berlin (2018)

Grigo, W., Heitfeld, M., Rosner, P., Welz, A.: Ein Konzept zur Überwachung der Auswirkungen des Grubenwasseranstiegs im Ruhrgebiet. In: Meier, G., Sroka, A., Löbel, K.-H., Klapperich, H., Tondera, D., Busch, W., Mass, K. (Hrsg.) 7. Altbergbau Kolloqium Freiberg. S. 250-269. (2007)

Grobe, M., Machel, H.G.: Saline groundwater in the Münsterland Cretaceous Basin, Germany: clues to its origin and evolution. Mar. Pet. Geol. 19(3), 307-322 (2002). https://doi.org/10.1016/S02648172(02)00019-3

Heim, S., Schwarzbauer, J., Kronimus, A., Littke, R., Woda, C., Mangini, A.: Geochronology of anthropogenic pollutants in riparian wetland sediments of the Lippe River (Germany). Org. Geochem. 35(11/12), 1409-1425 (2004). https://doi.org/10.1016/J. ORGGEOCHEM.2004.03.008

Heitfeld, K.H., Heitfeld, M., Rosner, P., Sahl, H., Schetelig, K.: Mine water recovery in the coal mining district of Aachen-impacts and measures to control potential risks. In: Merkel, B.J., PlanerFriedrich, B., Wolkersdorfer, C. (Hrsg.) Uranium in the Aquatic Environment, S. 1027-1036. Springer, Heidelberg (2002)

Herbert, H.-J., Sander, W.: Die Flutung des Kalibergwerks Hope Ergebnisse des geochemischen Meßprogramms. Kali Steinsalz 9(10), 326-333 (1987)

Himmelsbach, T., Wendland, E.: Schwermetalltransport in Sandsteinen unter Bedingungen einer hochsalinaren Porenwasserlösung - Laborversuch und Modellierung. Grundwasser 4(3), 103-112 (1999)

Hoth, K.: Die Steinkohlenlagerstätte Zwickau. Sächsisches Landesamt für Umwelt, Landwirtschaft und Geologie (LfULG), Freiberg (2008)

Jäger, B., Obermann, P., Wilke, F.L.: Studie zur Eignung von Steinkohlebergwerken im rechtsrheinischen Ruhrkohlenbezirk zur Untertageverbringung von Abfall-und Reststoffen. 4 Text-, 1 Anlagenband, unveröffentl. „Machbarkeitsstudie“ im Auftrag des Landesamt für Wasser und Abfall NRW, Düsseldorf (1990)

Jordan, I., Welsing, R.: Einstellung der Grubenwasserhaltung nach Beendigung der Steinkohlengewinnung. Z. Wasserr. 56(3), 121-141 (2017)

Kandola, B., Sullivan, M.: The use of bow-tie model and thesis in the control of major accident hazards. In: FABIG Technical Meeting (2003)

Kim, S.M., Choi, Y.: SIMPL: A simplified model-based program for the analysis and visualization of groundwater rebound in abandoned mines to prevent contamination of water and soils by acid mine drainage. Int. J. Environ. Res. Public Health 15(5), 951 (2018). https://doi.org/10.3390/ijerph15050951

Klinger, C., Charmoille, A., Bueno, J., Gzyl, G., Súcar, B.G.: Strategies for follow-up care and utilisation of closing and flooding in European hard coal mining areas. Int. J. Coal. Geol. 89, 51-61 (2012). https://doi.org/10.1016/J.COAL.2011.11.008

Klinger, C., Eckart, M., Charmoille, A., Le Gal, N., Bueno, J., Gzyl, G., Kura, K., Garzon, B.: Flooding management for underground coal mines considering regional mining networks (Flominet)—final report. Publications Office of the European Union, Luxemburg (2013) https://doi.org/10.2777/94695

Kories, H., Rüterkamp, P., Sippel, M.: Field and numerical studies of water stratification in flooded shafts. In: Jarvis, A.P., Dudgeon, B.A., Younger, P.L. (Hrsg.) International Mine Water Association 
Symposium 2004, S. 149-159. University of Newcastle, Newcastle upon Tyne (2004)

Kortas, L., Younger, P.L.: Using the GRAM model to reconstruct the important factors in historic groundwater rebound in part of the Durham Coalfield, UK. Mine Water Environ 26(2), 60-69 (2007). https://doi.org/10.1007/s10230-007-0152-8

Krause, E., Pokryszka, Z.: Investigations on methane emission from flooded workings of closed coal mines. J. Sustain. Min. 12(2), 40-45 (2013). https://doi.org/10.7424/jsm130206

Kretschmann, J., Efremenkov, A.B., Khoreshok, A.A.: From mining to post-mining: the sustainable development strategy of the German hard coal mining industry. Iop Conf. Ser. Earth Environ. Sci. 50(1), 12024 (2017). https://doi.org/10.1088/1755-1315/50/ $1 / 012024$

Krzemień, A., Suárez Sánchez, A., Riesgo Fernández, P., Zimmermann, K., González Coto, F.: Towards sustainability in underground coal mine closure contexts: a methodology proposal for environmental risk management. J Clean Prod 139, 1044-1056 (2016). https://doi.org/10.1016/j.jclepro.2016.08.149

Kurkamp, S., Pielka, B., Klingenburg, D.: Das Ende einer Epoche. Steinkohle (2019). Special Issue https://doi.org/10.14315/ 9783641248475-014

LAWA: Ableitung von Geringfügigkeitsschwellenwerten für das Grundwasser. LAWA, Düsseldorf, S. 33 (2004)

Luckner, L., Morgenstern, A.: Grundlagen von Schichtungsprozessen in gefluteten Untertagebergwerken. World Min. Surf. Undergr. 58(5), 311-315 (2006)

Lüders, V., Plessen, B., Romer, R.L., Weise, S.M., Banks, D.A., Hoth, P., Dulski, P., Schettler, G.: Chemistry and isotopic composition of Rotliegend and Upper Carboniferous formation waters from the North German Basin. Chem. Geol. 276(3/4), 198-208 (2010). https://doi.org/10.1016/J.CHEMGEO.2010.06.006

Melchers, C., Goerke-Mallet, P., Kleineberg, K.: Elemente und Aspekte des Nachbergbaus. Min. Rep. 152(3), 215-223 (2016)

Molson, J., Aubertin, M., Bussière, B.: Reactive transport modelling of acid mine drainage within discretely fractured porous media-Plume evolution from a surface source zone. Environ. Model. Softw. 38, 259-270 (2012). https://doi.org/10.1016/j. envsoft.2012.06.010

MUV, Ministerium für Umwelt und Verbraucherschutz: Hintergrundpapier Bergbau zum 2. Bewirtschaftungsplan 2016-2021 zur Umsetzung der EG-Wasserrahmenrichtlinie (2000/60/EG) im Saarland (2017)

Neymeyer, A., Williams, R.T., Younger, P.L.: Migration of polluted mine water in a public supply aquifer. Q. J. Eng. Geol. Hydrogeol. 40(1), 75-84 (2007). https://doi.org/10.1144/1470-9236/05-060

Niemann, A.: Raus aus der Grube - rein in die Grube: Ein untertägiges Pumpspeicherwerk als Bergbaunachfolge im Ruhrrevier. Herausford. Wasserforsch. Lokal Reg. Glob. 51, 106-115 (2018). https:// doi.org/10.17185/duepublico/70364

Niemann, A., Balmes, J.P., Schreiber, U., Wagner, H.-J., Friedrich, T. Proposed underground pumped hydro storage power plant at Prosper-Haniel colliery in Bottrop-state of play and prospects. Min. Rep. Glückauf 154(3), 214-223 (2018)

Niemann, A., Wortberg, T., Alvarado Montero, R.: Wasserbauliche und wasserwirtschaftliche Aspekte für die Umsetzung von Untertage-Pumpspeicherwerken in Anlagen des Steinkohlebergbaus. In: Perau, E. (Hrsg.) Untertage-Pumpspeicherwerke in Anlagen des Berg- und Tagebaus, S. 87-104. VGE, Essen (2013)

Nordstrom, D.K.: Mine waters: acidic to circumneutral. Elements 7(6), 393-398 (2011). https://doi.org/10.2113/gselements.7.6.393

Nordstrom, D.K.: Baseline and premining geochemical characterization of mined sites. Appl. Geochem. 57, 17-34 (2015). https:// doi.org/10.1016/J.APGEOCHEM.2014.12.010

Nordstrom, D.K., Alpers, C.N.: Geochemistry of acid mine waters. In: Plumlee, G.S., Logsdon, M.J. (Hrsg.) The Environmental Geochemistry of Mineral Deposits, S. 133-160. Society of Economic Geologists, Littleton (1999)
Nuttall, C.A., Younger, P.L.: Hydrochemical stratification in flooded underground mines: an overlooked pitfall. J. Contam. Hydrol. 69(1/2), 101-114 (2004). https://doi.org/10.1016/S01697722(03)00152-9

OGewV: Oberflächengewässerverordnung vom 20. Juni 2016. BGB1. I (2016)

Pauwels, H., Fouillac, C., Fouillac, A.-M.: Chemistry and isotopes of deep geothermal saline fluids in the Upper Rhine Graben-Origin of compounds and water-rock interactions. Geochim. Cosmochim. Acta 57(12), 2737-2749 (1993). https://doi.org/10.1016/ 0016-7037(93)90387-C

Perau, E., Schreiber, U., Niemann, A., Koch, K.: Unterflur-Pumpspeicherwerke in Anlagen des Steinkohlebergbaus. Schriftenr. Tech. Univ. Zent. Geotech. München 52, 27-38 (2012)

Primrose, M.J., Bentley, P.D., van der Graaf, G.C., Sykes, R.M.: The HSE management system in practice-implementation. In: SPE Health, Safety and Environment in Oil and Gas Exploration and Production Conference. Society of Petroleum Engineers, New Orleans, Louisiana (1996)

RAG: Bau einer Wasserstoffperoxidanlage zur Behandlung des sulfidhaltigen Grubenwassers der Wasserhaltung Camphausen (2010)

RAG: Konzept zur langfristigen Optimierung der Grubenwasserhaltung der RAG Aktiengesellschaft in Nordrhein-Westfalen, Herne (2014). http://www.bid.rag.de/bid/PDFs/GWA/Konzept_ Grubenwasserhaltung.pdf. Zugegriffen: 17.08.2020

Rahm, H.: Belastungen von Oberflächengewässern und von aktiven Grubenwassereinleitungen mit bergbaubürtigen PCB (und PCBErsatzstoffen). Landesamt für Natur, Umwelt und Verbraucherschutz NRW, Recklinghausen (2018)

Reason, J.T.: Human Error. Cambridge University Press, Cambridge (1990)

Reason, J.T.: Managing the Risks of Organizational Accidents. Routledge, Aldershot (1997)

Reiners, T., Wagner, H.-J., Penczek, N., Altieri, L., Gross, M.: Klimafreundliches Heizen durch Wärme aus Grubenwasser im Nachbergbau. Min. Rep. Glückauf 154(3), 200-208 (2018)

Röder, U.: Geothermische Nutzung von Flutungswässern aus den Abbauhohlräumen des Zwickauer Steinkohlenreviers: Schlussbericht. Staatsbetrieb Sächsisches Immobilien- und Baumanagement, NL Zwickau, Zwickau, S. 1275 (2018)

Rosner, P.: Der Grubenwasseranstieg im Aachener und Südlimburger Steinkohlenrevier - Eine hydrogeologisch-bergbauliche Analyse der Wirkungszusammenhänge. RWTH, Aachen (2011)

Rosner, P., Heitfeld, M., Spreckels, V., Vosen, P.: Auswirkungen von Geländehebungen im Zuge des Grubenwasseranstiegs im Ruhrrevier. In: Tagungsband RuhrGeo Tag Bochum. S. 153-177. (2014)

Rüterkamp, P.: Bildung von Dichteschichtungen in Grubenwässern. Glückauf-Forschungsh. 62(2), 40-44 (2001)

Sächsisches Landesamt für Umwelt, Landwirtschaft und Geologie: Mobile Wasserbehandlungsanlage zur Enteisenung und Entmanganung von Grubenwasser aus Tiefbohrung Oelsnitz (2018). http://www.vitamin-projekt.eu/sanierungsverfahren-3969.html. Zugegriffen: 17.08.2020

Sherwood, J.M., Younger, P.L.: Modelling groundwater rebound after coalfield closure-An example from County Durham, United Kingdom. In: Proceedings of the 5th International Minewater Congress Nottingham, UK. S. 769-777. (1994)

Singer, P.C., Stumm, W.: Acidic mine drainage: the rate-determining step. Science 167(3921), 1121-1123 (1970). https://doi.org/10. 1126/science.167.3921.1121

Stuart, W.T., Simpson, T.A.: Variations of $\mathrm{pH}$ with depth in anthracite mine-water pools in Pennsylvania; Article 37. U. S. Geol. Surv. Prof. Pap. 0424-B, B82-B84 (1961)

Stumm, W., Morgan, J.J.: Aquatic Chemistry-Chemical Equilibria and Rates in Natural Waters, 3. Aufl. Wiley, New York (1996) 
Toran, L., Bradbury, K.R.: Ground-water flow model of drawdown and recovery near an underground mine. Ground Water 26(6), 724-733 (1988)

Wagner, J.: Fachgutachtliche hydrogeologische Beurteilung des Grubenwasseranstiegs in bergbaubedingten untertägigen Hohlräumen nach Einstellen des Kohleabbaus im Saarkarbon. ProjektAbschlussbericht. Oberbergamt des Saarlandes, Neunkirchen (2017)

Wanner, C., Eichinger, F., Jahrfeld, T., Diamond, L.W.: Causes of abundant calcite scaling in geothermal wells in the Bavarian Molasse Basin, Southern Germany. Geothermics 70, 324-338 (2017). https://doi.org/10.1016/j.geothermics.2017.05.001

Wedewardt, M.: Hydrochemie und Genese der Tiefenwässer im RuhrRevier. DMT-Berichte aus Forschung und Entwicklung, Bd. 39. (1995)

Westermann, S., Dogan, T., Reker, B., Goerke-Mallet, P., Wolkersdorfer, C., Melchers, C.: Evaluation of mine water rebound processes in European coal mine districts to enhance the understanding of hydraulic, hydrochemical and geomechanical processes. In: Wolkersdorfer, C., Sartz, L., Sillanpää, M., Häkkinen, A. (Hrsg.) IMWA 2017-Mine Water \& Circular Economy I, S. 147-153. Lappeenranta University of Technology, Lappeenranta (2017)

WHG: Wasserhaushaltsgesetz vom 31. Juli 2009. BgB1. I (2009)

Wieber, G.: Stillgelegte geflutete Erzbergwerke - schädliche Bodenveränderungen gemäß Bundes-Bodenschutzgesetz? Altlasten Spektrum 5, 201-209 (2013)

Witthaus, H., Drobniewski, M.: Monitoring of mine water. In: Wolkersdorfer, C., Sartz, L., Sillanpää, M., Häkkinen, A. (Hrsg.) IMWA 2017-Mine Water \& Circular Economy, S. 88-93. Lappeenranta University of Technology, Lappeenranta (2017)

Wohnlich, S., Stemke, M., Wisotzky, F., Englert, A., Banning, A., Eckart, M., Gatta, T., Knüfermann, C., Knuth, C.: Entwicklung eines Realisierungskonzeptes für die Nutzung von Anlagen des Steinkohlenbergbaus als unterirdische Pumpspeicherkraftwerke (2013). http://upsw.de/files/artikelbilder/Dokumente_und_
Screenshots_28.01.2014/20160822_UPSW_Zusammenfassung. pdf. Zugegriffen: 17.08.2020

Wolkersdorfer, C.: Hydrogeochemische Verhältnisse im Flutungswasser eines Uranbergwerks - Die Lagerstätte Niederschlema/ Alberoda. Clausthal. Geowiss. Diss. 50, 1-216 (1996)

Wolkersdorfer, C.: Water Management at Abandoned Flooded Underground Mines-Fundamentals, Tracer Tests, Modelling, Water Treatment. Springer, Heidelberg (2008)

Wolkersdorfer, C.: Grubenwasserreinigung - Beschreibung und Bewertung von Verfahren. Springer, Heidelberg (2020)

Wolkersdorfer, C., Bantele, M.: Die Oberbayerischen Pechkohlenmulde - Hydrogeochemische Untersuchungen der Grubenwässer. Grundwasser 18(3), 185-196 (2013)

Wolkersdorfer, C., Bowell, R.J.: Contemporary reviews of mine water studies in Europe. Mine Water Environ. 24(3), 1-76 (2005)

WRR: Richtlinie 2000/60/EG des Europäischen Parlamentes und des Rates zur Schaffung eines Ordnungsrahmens für Maßnahmen der Gemeinschaft im Bereich der Wasserpolitik. Europäische Kommission, Brüssel (2000)

Yang, Y., Ligouis, B., Pies, C., Achten, C., Hofmann, T.: Identification of carbonaceous geosorbents for PAHs by organic petrography in river floodplain soils. Chemosphere 71(11), 2158-2167 (2008). https://doi.org/10.1016/J.CHEMOSPHERE.2008.01.010

Younger, P.L.: The longevity of minewater pollution: a basis for decision-making. Sci. Total Environ. 194/195, 457-466 (1997). https:// doi.org/10.1016/S0048-9697(96)05383-1

Younger, P.L., Adams, R.: Predicting Mine Water Rebound. Environmental Agency, Bristol (1999)

Younger, P.L., Banwart, S.A., Hedin, R.S.: Mine Water-Hydrology, Pollution, Remediation. Kluwer, Dordrecht (2002)

Hinweis des Verlags Der Verlag bleibt in Hinblick auf geografische Zuordnungen und Gebietsbezeichnungen in veröffentlichten Karten und Institutsadressen neutral. 